\title{
ESTIGMATIZACIÓN A PERSONAS CON VIH-SIDA EN LA ATENCIÓN PRIMARIA COMO BARRERA DE APEGO AL TRATAMIENTO.
}

\author{
STIGMATIZATION OF PEOPLE WITH HIV-AIDS IN PRIMARY CARE AS A BARRIER ADHERENCE TO THE \\ TREATMENT
}

Saucedo Pahua Gerardo ${ }^{1-2}$, Huerta Baltazar Mayra Itzel ${ }^{1}$, Alcántar Zavala Ma. Lilia ${ }^{1-3}$, Ruiz Recéndiz Ma. de Jesús ${ }^{1}$, Jiménez Arroyo Vanesa ${ }^{1}$, Avila Cazarez Lorena ${ }^{1-2}$

1 Universidad Michoacana de San Nicolás de Hidalgo, México. 2 Hospital General Regional №. 1 del Instituto Mexicano del Seguro Social, Morelia, Michoacán, México. 3 Hospital Infantil "Eva Sámano de López Mateos", México.

Citation: Saucedo Pahua G., Huerta Baltazar M.I., Alcántar Zavala M.L., Ruiz Recéndiz M.J., Jiménez Arroyo V., Avila Cazarez L. (2018) Estigmatización a personas con VIH-SIDA en la atención primaria como barrera de apego al tratamiento. Revista de Salud Pública y Nutrición, 17(3), 26-33.

Editor: Esteban G. Ramos Peña, Dr. CS., Universidad Autónoma de Nuevo León, Facultad de Salud Pública y Nutrición, Monterrey Nuevo León, México. Copyright: (C2018 Saucedo Pahua G. et al. This is an open-access article distributed under the terms of Creative Commons Attribution License [CC BY 4.0], which permits unrestricted use, distribution, and reproduction in any medium, provided the original author and source are credited.

Competing interests: The authors have declared that no competing interests exist.

DOI: https://doi.org/10.29105/respyn17.3-4

Recibido: 13 de agosto 2018;

Aceptado: 24 de septiembre 2018

Email: ge_ra_sol@hotmail.com 


\title{
ESTIGMATIZACIÓN A PERSONAS CON VIH-SIDA EN LA ATENCIÓN PRIMARIA COMO BARRERA DE APEGO AL TRATAMIENTO
}

\author{
Saucedo Pahua Gerardo ${ }^{1-2}$, Huerta Baltazar Mayra Itzel ${ }^{1}$, Alcántar Zavala Ma. Lilia ${ }^{1-3}$, Ruiz Recéndiz Ma. de \\ Jesús ${ }^{1}$, Jiménez Arroyo Vanesa ${ }^{1}$, Avila Cazarez Lorena ${ }^{1-2}$
}

1 Universidad Michoacana de San Nicolás de Hidalgo. 2 Hospital General Regional №. 1 del Instituto Mexicano del Seguro Social, Morelia, Michoacán. 3 Hospital Infantil "Eva Sámano de López Mateos".

\section{RESUMEN}

Introducción: La infección por VIH es un problema a nivel mundial que afecta la salud de quien la contrae y en él se experimentan procesos psicoemocionales y de estigmatización. El estigma, es resultado del desconocimiento de los actores directos en la atención primaria a la salud. Este problema social crea una barrera en las personas seropositivas a no buscar un tratamiento oportuno, lo que deteriora ampliamente su calidad de vida. Objetivo: Presentar una reflexión crítica sobre las razones por el cual las personas seropositivas refieren sentir estigmatización y discriminación por los involucrados en la atención primaria. Conclusiones: Dentro de la cultura Mexicana existe una diversidad de pensamiento hacia las personas que viven con VIH, dado a esto se han implementado políticas públicas a favor de éste sector altamente vulnerable, el propósito es salvaguardar y respetar la integridad de los individuos seropositivos, del mismo modo la Secretaría de Salud es el eje rector en promover información, detección y tratamiento oportuno a las personas que demandan atención primaria a la salud, mediante la difusión de información científica a través de los actores directos de la atención. Es necesario desarrollar herramientas de evaluación que permitan corroborar que la información emitida tenga un impacto cognitivo significativo. Palabras Clave: VIH-SIDA, Estigma y discriminación, Atención Primaria.

\section{ABSTRACT}

Introduction: HIV infection is a worldwide problem that affects the health of those who contract it and in it psycho-emotional and stigmatization processes are experienced. The stigma is the result of ignorance of the direct actors in primary health care. This social problem creates a barrier in seropositive people not to seek timely treatment, which greatly deteriorates their quality of life. Objective: To present a critical reflection on the reasons why HIV-positive people report feeling stigmatization and discrimination for those involved in primary care. Conclusions: Within the Mexican culture there is a diversity of thinking towards people living with HIV, given that public policies have been implemented in favor of this highly vulnerable sector, the purpose is to safeguard and respect the integrity of HIV-positive individuals. Similarly, the Ministry of Health is the guiding axis in promoting information, detection and timely treatment for people who demand primary health care, through the dissemination of scientific information through the direct actors of care. It is necessary to develop assessment tools to corroborate that the information issued has a significant cognitive impact.

Key words: HIV-AIDS, Stigma and discrimination, Primary Care. 


\section{Introducción}

La pandemia del virus de inmunodeficiencia humana y el síndrome de inmunodeficiencia adquirida (VIHSIDA) han sido un problema de salud pública a nivel mundial (Compean, Pérez, Staines y Ortiz, 2014). Según el informe del Programa Conjunto de Naciones Unidas sobre el VIH/SIDA (ONUSIDA) a fínales de 2015, 36.7 millones de personas vivían con el virus en mundo (ONUSIDA, 2016) y México no es la excepción, con 24,718 casos de VIH-Sida, predominando en jóvenes de 15 y 29 años de edad (CENSIDA, 2015).

El VIH es un problema de salud que abarca esferas psicológicas y emocionales, a nivel personal y social; El estigma aparece como un atributo desacreditante en las personas seropositivas, como barrera a no buscar tratamiento, generando preocupación, ansiedad y depresión, deteriorando su calidad de vida (CV) (Pantoja y Estrada, 2014).

Desde 1994 Forstein refiere que, las personas sienten estigmatización por parte de profesionales de la salud, al momento de solicitar el cribado de VIH y para acceder a servicios de salud una vez que han sido diagnosticados seropositivos. (Varas, 2008); aunado a esto en 2003 Infante et al., refieren que en México las PVVIH refieren una mala calidad de atención por parte de los prestadores de servicios de salud.

Por otra parte, Chong et al., 2012 refieren que, las personas seropositivas hombres y mujeres heterosexuales y hombres que tienen sexo con hombres (HSH) atendidos en un hospital público del estado de Chiapas, mantienen oculto el diagnóstico, por miedo al estigma por la comunidad donde residen. Al expresar que les es difícil enfrentar el estigma y discriminación que emana los profesionales de la salud al momento de la atención en salud para su control.

Dado lo anterior en América Latina y el Caribe es casi nula la evidencia respecto a las políticas públicas en materia de salud y de derechos humanos, respecto a programas holísticos libres de discriminación hacia las PVVIH; en el caso de Colombia, este país en1988 elaboró el primer Plan Nacional de Prevención y Control del SIDA, estableciendo en 1991 por primera vez normas sobre la atención integral, derechos y deberes de las PVVIH (Arrivillaga et al., 2009)., entre éste periodo Costa Rica, República Dominicana, Ecuador, Guatemala, Honduras, Nicaragua, panamá y Puerto Rico decretaron leyes en los que abordan el tema de VIH-SIDA, en materia de prevención, tratamiento, derechos y responsabilidades de las personas seropositivas (Varas y Toro, 2003).

Torres (2006) realizó un análisis en relación a las políticas públicas en salud en respuesta al VIH-SIDA en México donde concluye que éstas han beneficiado a grupos que han sido tradicionalmente marginados y excluidos del proceso de implementación de las mismas; en 2015 la investigadora Rangel en su manuscrito detalla los procesos políticos de trasformación a la Ley en salud y de derechos humanos en la Republica Mexicana, dado a que la enfermedad ha formado parte de la agenda como problema de salud pública; en este mismo años los investigadores Orozco et al., realizaron un mapeo político y revisión del marco normativo para la prevención del VIH y fortalecer las acciones contra el estigma, la discriminación y la homofobia por parte de los involucrados en la atención en salud.

Bermúdez et al., 2015; argumentan que los profesionales de la salud tienen la responsabilidad y el compromiso de brindar una atención de calidad libre de estigma y discriminación hacia las personas que viven con virus de inmunodeficiencia humana (PVVIH); dado a que el objetivo en materia de salud pública es educar, prevenir, diagnosticar y otorgar un tratamiento oportuno y a su vez concientizar a la PVVIH a hacer adherencia a la terapia antirretroviral (TARV) en el éxito del tratamiento al VIH (Oliva, Lastre, Viñas y González, 2013). Cualquier actitud estigmatizante por parte de los mismos tiene un impacto psicoemocional, en la vida de las personas seropositivas (Pantoja y Estrada, 2014).

Éste ensayo tiene como propósito presentar una reflexión crítica sobre las razones por el que las personas seropositivas refieren sentir estigmatización y discriminación por los involucrados en la atención primaria. Para ello se discute sobre el papel de las políticas públicas en salud y de derechos humanos para sustentar una atención medica libre de discriminación, en la PVVIH. 
La metodología empleada a este manuscrito fue; una revisión sistemática en español e inglés en diez bases de datos electrónicas en salud, las cuales fueron: PubMed, EBSCO, Scielo, CUIDEN, Aquichan, Redalyc, RESPYN, DOAJ - Directory of Open Access Journals, Free Medical Journals y Google académico, Se utilizaron los siguientes términos de búsqueda: VIH-SIDA, Estigma y discriminación, Atención primaria a la salud, Políticas Publicas, Derechos humanos.

Los criterios de selección fueron; 1) trabajos de enero de 2013 a abril de 2018 2) trabajos en los cuales se analizaba la vulnerabilidad al VIH de forma específica y clara en relación al estigma y discriminación que viven las personas con VIH por parte de los prestadores de servicios en salud en el primer nivel de atención.3) trabajos que empleaban un enfoque desde las políticas públicas en salud y de derechos humanos respecto al VIH-SIDA y 4) trabajos cualitativos que expresaran las barreras de apego al tratamiento retroviral relacionado a la estigmatización percibida.

De 109 resultados, 35 investigaciones de tipo cuantitativo y cualitativo con diseños descriptivos y correlacional cumplieron los criterios de inclusión y de evaluación para su análisis y discusión de la información para el presente ensayo; el resto de la evidencia científica fue omitida dado a que no cumplieron los criterios de selección.

A la luz de los resultados y del análisis se presenta a continuación la respuesta al objetivo del presente manuscrito. En el primer apartado se describe el conocimiento, las habilidades y la actitud del personal de salud sobre VIH-Sida en la atención primaria a la salud, en seguida se presenta la evidencia científica que describe la estigmatización y discriminación como barrera de apego al tratamiento en las PVVIH, seguida de esto se muestran las políticas públicas dirigidas a la atención medica libre de discriminación, a personas que viven con VIH-Sida en México y se finaliza con las conclusiones.

Bajo este eje temático, es importante resaltar la relevancia sobre la adquisición de conocimientos que todos los profesionales de salud deben tener en relación a: la infección del VIH y SIDA, mediante políticas públicas en salud y de derechos humanos; dado a la necesidad apremiante que existe de fortalecer la educación formal de los profesionales futuros en estos tres ejes de conocimiento; en virtud de que el desconocimiento de los involucrados afecta gravemente al estado psícoemocional de la persona seropositiva. Del mismo modo se presentan estudios realizados en las PVVIH, en el que muestran sentirse vulnerables por el estigma y la discriminación de los actores en la atención primaria a la salud, lo que ocasiona una barrera al apego terapéutico.

Conocimiento, habilidad y actitud del personal de salud sobre VIH-SIDA en la atención primaria a la $\underline{\text { salud }}$

En 1980, inició la epidemia del VIH-SIDA a nivel mundial, y por consecuente la propagación de información formal e informal, que ha sido evaluada con aspectos asociados al conocimiento, aptitudes y prácticas relacionadas a la infección por el VIH, a grupos profesionales de la salud involucrados en la asistencia a personas infectadas por el virus (Barbosa et al, 2015). De acuerdo a Bolaños en 2015, recomienda que el equipo básico de salud (EBS), debe estar preparado con los conocimientos, habilidades y actitudes suficientes en la prevención VIH/SIDA, ante el riesgo que presenta la comunidad (Bolaños et al., 2015).

En 1996 se obtuvo el acceso y utilización a la terapia antirretroviral (TARV), lo que ha permitido una importante reducción de la morbi-mortalidad por el virus, en las PVVIH (Gilmara et al., 2015); dicha adherencia ha aumentado la calidad de vida en años en las personas seropositivas, quienes experimentan procesos complejos de transformación personal al vivir con una enfermedad crónica (Hipólito et al., 2017); por tal motivo los profesionales de salud deben poseer dominio sobre las consideraciones biológicas, psicológicas y sociales, para proporcionar cuidados holísticos a las personas seropositivas, con la mayor ética profesional (Gómez et al., 2013).

Como profesionales de salud, se debe estar en total disposición para prestar atención primaria, promoviendo el más alto grado de bienestar al paciente, garantizando su actuar en la atención integral del usuario (Bermúdez et al., 2017). En el actuar del equipo multidisciplinario, los profesionales de salud directos en observar los avances terapéuticos, es Enfermería, quien participa 
en la detección, cuidado y control de la enfermedad crónica (Costa et al., 2012).

El control clínico en las PVVIH, actualmente es una herramienta indispensable para el acompañamiento de los avances y esfuerzos necesarios para la mejoría en la atención, en el aumento y la demanda de la atención primaria a la salud (APS) (Leite et al., 2017).

En 2014, Carvalho et al., en su estudio: "Valores intervinientes en el cuidado del enfermero al cliente con VIH/Sida", concluyen que cada miembro del equipo de salud debe autoconocerse, lo que implica la reflexión de sus valores, creencias y cultura, con el fin de que éstos no interfieran negativamente en la realización de sus acciones de atención primaria a la salud.

De esta manera, los profesionales de salud son considerados un pilar fundamental en la oferta del tamizaje en la APS, y el desconocimiento de ello limita la calidad del servicio a este grupo vulnerable (Pantoja, 2014). De tal manera que el rechazo y la discriminación percibido por el usuario durante la detección, evita buscar un tratamiento y adherencia al mismo, por miedo a la estigmatización ya vivenciada, lo que limita ampliamente su CV (Cunha et al., 2015).

Evidencia científica de la estigmatización y discriminación como barrera de apego al tratamiento en las PVVIH

A lo largo de muchas décadas, la estigmatización ha sido reconocida como una conducta inmoral, descrito por Erving Goffman y Herek, atributo que desacredita al individuo y tiene un efecto indeseable en la condición humana, (Erving Goffman y Herek, citado por Pantoja et al., 2014). Colbert et al., definen al estigma como un proceso social relacionado con la salud, que es vivenciado, anticipado, caracterizado por exclusión, rechazado, con sensaciones de culpa y/o devaluación; que resulta de la experiencia de una persona o grupo.

En la actualidad existen mitos y creencias relacionados con el VIH/SIDA, que conducen a prácticas de estigma social hacia las personas seropositivas, de acuerdo a la evidencia científica la infección del VIH se asocia directamente a conductas de alto riesgo, y por ende, esto altera el comportamiento de los profesionales de la salud que se manifiestan en prácticas estigmatizadoras y discriminativas dirigidas con juicios negativos sobre su moral, conductas irrespetuosas e incluso dominadas al temor a contraer el virus (Barbosa, 2015).

La credibilidad de los profesionales de salud se ve afectada por aspectos como la desinformación y estereotipos sociales, lo que genera actitudes estigmatizantes, que son percibidas por las personas seropositivas (Pantoja, 2014); lo que minimiza el éxito en el apego al tratamiento, estas dificultades de acceso a los servicios de salud aumentan el riesgo de aumentar la carga viral en las personas con VIH, reduciendo la CV en la persona (Leite, 2017).

Tamayo et al., 2015 en su estudio "Estigma social en la atención de personas con $\mathrm{VIH} /$ sida por estudiantes y profesionales de las áreas de la salud, en Medellín, Colombia" afirman que el estigma por parte del personal de la salud tiene grandes implicaciones en la calidad del servicio el cual hace que las personas VIH positivas no acudan oportunamente a los servicios de salud; estos hallazgos se relacionan a lo encontrado por Bermúdez et al., en 2015, donde afirma que el estigma y la discriminación alrededor del VIH construye una barrera que impide el acceso a los servicios de salud. En definitiva, los investigadores consideran relevante evitar la discriminación en la prestación de servicios de salud.

Guardia et al., 2015 en su investigación presentan las narrativas de PVVIH en situaciones discriminatorias que ellos atribuyen al sistema de salud y a la bioseguridad. Los autores concluyen desde su perspectiva que las personas confunden las características estructurales del sistema de salud con estigmatización; sin embargo, en el estudio los trabajadores de la salud ponen al descubierto sus temores irracionales al contagio.

Scher (2016) refiere en su estudio que, cada participante ha percibido algún tipo de estigma, y también ha recibido algún tipo de discriminación durante su vida en distintos grados., por tanto, se revelaron experiencias de discriminación en el ámbito escolar, familiar, laboral, y en la vida cotidiana de las PVVIH., cada mujer trans se refirió al hecho de que la mayoría de ellas trabajan en el 
comercio sexual debido a las condiciones de estigma y discriminación que enfrentan en el día a día.

De igual modo, Fuster et al., en 2013 elaboraron un cuestionario para medir las estrategias de las personas con VIH para afrontar el estigma. Los resultados arrojados mostraron que las personas con VIH utilizan una variedad de estrategias de afrontamiento que se sitúan en el control primario, así como en el secundario., éstas, abren el panorama de intervenciones para capacitar a las personas con VIH y enfrentar el estigma y la discriminación.

Políticas públicas dirigidas a la atención medica libre de discriminación, a personas que viven con VIHSIDA en México

Dentro de la Constitución Política de los Estados Unidos Mexicanos el Artículo $1^{\circ}$ tercer párrafo establece que: queda prohibida toda discriminación hacia las condiciones de salud y preferencias sexuales de las personas, esto aunado al artículo $4^{\circ}$ tercer párrafo, donde se afirma que: toda persona tiene derecho a la protección de la salud, y establece que la ley definirá las bases y modalidades para el acceso a los servicios de salud (Constitución Política de los Estados Unidos Mexicanos, 1917).

En este mismo país las acciones para la prevención, detección y diagnóstico oportuno de las infecciones de trasmisión sexual (ITS) y de VIH-SIDA son prioritarias en el sistema de salud y se sustentan en normas y políticas públicas específicas en el Programa Sectorial de Salud 2013-2018, (2013). Así mismo, se describe en la presentación del Programa de Acción Específico Respuesta al VIH, Sida e ITS, tras el decreto de otorgar tratamiento oportuno a estos problemas de salud pública, de forma gratuita y universal a personas altamente vulnerables, siendo el factor más importante en la reducción de la morbimortalidad de la infección por el VIH.

La Ley General de Salud se aplica en toda la República Mexicana y sus disposiciones son de orden público e interés social, en lo concerniente a la detección oportuna de las ITS y de VIH-SIDA la Norma Oficial Mexicana NOM-010-SSA2-2010. Para la prevención y control de la infección por virus de la inmunodeficiencia humana, tiene un marco de confidencialidad, siendo una disposición de observancia obligatoria en el territorio nacional para todas las instituciones y personal del Sistema
Nacional de Salud involucrado en la atención a las PVVIH.

En 1990 se creó la comisión Nacional de los Derechos Humanos (CNDH) la cual ha trabajado activamente tras las violaciones a los derechos humanos que han presentado de forma oficial las PVVIH en la negación o inadecuada prestación del servicio público de salud (CNDH, 2015); Trece años después, el Diario Oficial de la Federación (DOF) publica en 2003 la Ley Federal para prevenir y eliminar la discriminación (LFPED), la cual establece que, queda prohibida toda práctica discriminatoria que tenga por objeto o efecto impedir $\mathrm{o}$ anular el reconocimiento o ejercicio de los derechos e igualdad, creando para ello, el Consejo Nacional para Prevenir la Discriminación (CONAPRED), el cual es el órgano encargado de promover políticas públicas, garantizando el derecho a la igualdad e inclusión social.

A la luz de las políticas públicas anteriormente mencionadas se ha creado un pliego de derechos humanos hacia las personas que viven con VIH o con SIDA, en el que se establece la igualdad de derecho a vivir sin discriminación o violación a las garantías individuales y colectivas en la persona o de quien dependa de ellos (CNDH, 2016), en el entendido que todos los profesionales de la atención primaria están comprometidos a brindar atención holística y pandimensional, tal como lo refiere Betty Newman en su teoría.

\section{Conclusiones}

En conclusión, las PVVIH manifiestan que han experimentado de estigmatización y discriminación, tras esta experiencia las personas seropositivas expresan la necesidad de sentir apoyo emocional en especial de su familia y de su red social, lo que les facilita la búsqueda de información y tratamiento farmacológico, situación inherente a la aceptación de esta enfermedad crónica.

Igualmente se encontró que las PVVIH cuentan con información entorno a su enfermedad, puesto que el alcance a la tecnología ha permitido que las personas consulten páginas de internet $\mathrm{e}$ integre este conocimiento a su contexto en particular para construir una visión clara de su propio riesgo y las alternativas que tienen para reducirlo. Justamente estos hallazgos exponen la relevancia de mejorar los 
procesos de la educación formal entre los profesionales de la salud de todos los niveles de atención, respecto a la información correcta de la enfermedad, tratamiento y control, con base en la evidencia científica actual.

La cultura mexicana, pues, emerge de una diversidad de pensamientos y actos reprobados hacia las PVVIH, y por tal motivo se ha trabajado en establecer y practicar las políticas públicas, en los programas de "asistencia social" a PVVIH, ya que la problemática de personas que viven con VIH/SIDA en el mundo ha aumentado en gran medida y ha causado estragos sociales, económicos, culturales y problemas dentro del núcleo familiar. Por lo anterior México, tiene políticas públicas a favor de las PVVIH, que se sustentan en el Programa de Acción Específico 2013 -2018 - Respuesta al VIH, SIDA e ITS, cuyo objetivo prioritario es la prevención, detección y control de las personas altamente vulnerables, mediante políticas y estrategias que hacen frente a esta problemática en el país.

En esta misma línea de ideas, el acceso a los servicios de APS libres de discriminación son una determinante importante para el tamizaje oportuno, diagnostico asertivo para el tratamiento efectivo en la limitación del daño, que permita eliminar las barreras institucionales de acceso a la atención y al uso de los servicios sin temor a experimentar estigmatización por parte de los involucrados en la atención primaria a la salud; por ello, en ésta se plantean grandes desafíos en la gestión de servicios de salud para los diversos actores del sistema, en enfocar la atención a grupos vulnerables, y se superan barreras de acceso a los servicios de salud si los involucrados conocen las políticas públicas en relación a una tención libre de discriminación, como derecho hacia las PVVIH.

Finalmente, conviene resaltar que para difundir los conocimientos en salud es imprescindible, y al mismo tiempo es necesario desarrollar herramientas de evaluación que permitan corroborar que la información proporcionada a los profesionales de la salud y las PVVIH ha sido comprendida y en su defecto, replantear nuevas técnicas de aprendizaje para lograr un conocimiento cognitivo significativo.

\section{Bibliografía}

Arrivillaga, M., Alzate, M.L y Useche, B. (2009). Políticas públicas, sistema de salud y mujeres con VIH/SIDA en Colombia: un análisis crítico. Revista Gerencia y Políticas de Salud. 8(16); 57-71. Recuperado de: http://www.redalyc.org/articulo.oa?id=54514071004

Barbosa, B., Guimarães, J., Salge, A.K y Fávaro, C. (2015 Julio). Conocimiento de los profesionales de la salud en la prevención de la transmisión vertical del VIH en una maternidad pública brasileña. Enf. Global, 39,1$14 . \quad$ Recuperado de: http://scielo.isciii.es/scielo.php?script=sci_arttext\&pi $\mathrm{d}=\mathrm{S} 169561412015000300001$

Bermúdez, V., Bran, L., Palacios, L y Posada, I.C. (2015). Influencia del estigma en torno al VIH en el acceso a los servicios de salud. Salud Publica Mex. 57; 252-259. Recuperado de: http://www.redalyc.org/articulo.oa?id=10638801008

Bermúdez, V., Bran, L., Palacios, L y Posada, I.C. (2017). Relación médico-paciente: impacto en las campañas de promoción y prevención para personas con VIH en Medellín. Rev. Salud pública. 18(4); 543-553, 2874. Recuperado

de: http://www.redalyc.org/articulo.oa?id=42247581004

Bolaños, M.R., Díaz. M y Segredo, A. (2015). Curso para el equipo básico de salud sobre el control y prevención del VIH/sida. Educación Médica Superior. 29 (2); 273 $279 . \quad$ Recuperado de: http://scielo.sld.cu/scielo.php?script=sci_arttext\&pid= $\underline{\text { S086421412015000200008 }}$

Carvalho, F y Carvalho, C. (2014). Valores intervinientes en el cuidado del enfermero al cliente con VIH/Sida. Aquichan. 14(1); 32-40. Recuperado de: http://aquichan.unisabana.edu.co/index.php/aquichan/ article/view/3525/html

Chong, F., Fernández, C., Huicochea, L., Álvarez, G y Leyva, R. (2012). Estrategias de personas con VIH para enfrentar el estigma asociado al VIH / sida. Pacientes del Hospital General de Huixtla, Chiapas. Rev. Estudios Fronterizos. 13 (25); 31-55. Recuperado de:

http://www.redalyc.org/articulo.oa?id=53023332002

Comisión Nacional de los Derechos Humanos. (2016). Derechos humanos de las personas que viven con VIH $o$ con sida. México. Recuperado de: http://www.cndh.org.mx/sites/all/doc/cartillas/1_Carti $\underline{\text { lla_VIH_sida.pdf }}$

Comisión Nacional de los Derechos Humanos. (2016). Recomendaciones de la $\mathrm{CNDH}$ relacionadas con el 
VIH y el Sida. Recuperado de: http://appweb.cndh.org.mx/biblioteca/archivos/pdfs/li b_RecomendacionesCNDH_VIHSida.pdf

Compean, M.S., Pérez, D., Staines, M.G y Ortiz, L. (2014). Factores sociodemográficos asociados a la satisfacción con los servicios públicos de salud entre personas con vih/sida. Rev. Gerenc. Polit. Salud.13 (26); 127-143. Recuperado de: http://www.scielo.org.co/pdf/rgps/v13n26/v13n26a10. pdf

Constitución Política de los Estados Unidos Mexicanos. DOF 05-02-1917 Ref. Última reforma publicada DOF 15-09-2017: 2-8. Recuperado de: http://www.diputados.gob.mx/LeyesBiblio/pdf/1 150 917.pdf

Costa, T.L. Oliveira, D.C. Formozo, G.A y Gomes, A.M.T. (2012). Personas con HIV/SIDA en las representaciones sociales de enfermeros: análisis de los elementos centrales, contra normativos y actitudinales. Rev. Latino-Am. Enfermagem. 20(6); 1-10. Recuperado http://www.scielo.br/pdf/rlae/v20n6/es_11.pdf

Cunha, G.H., Teles, M.L., Gir, E., Souza, P., Bezerra, A.K., Gimeniz, M.T.(2015). Calidad de vida de hombres con Sida y el modelo de la determinación social de la salud. Rev. Latino-Am. Enfermagem. 23(2)::183-91. Recuperado de: http://www.scielo.br/pdf/rlae/v23n2/es 0104-1169rlae-23-02-00183.pdf

Fuster, M.J., Molero, F., Holgado, F.P y Sansinenea, E. (2013). Desarrollo de un nuevo Cuestionario para Medir las Estrategias que las Personas con VIH Usan para Afrontar el Estig-ma y la Discriminación. Acción Psicológica. 10(2); 3-20. Recuperado de: http://scielo.isciii.es/pdf/acp/v10n2/08monografico8.p df

Gilmara, G., Teles, M.L., Gir, E., Souza, P., Bezerra, AK y Gimeniz, M.T.(2015). Calidad de vida de hombres con Sida y el modelo de la determinación social de la salud. Rev. Latino-Am. Enfermagem. 23(2); 183-91. Recuperado de: http://www.scielo.br/pdf/rlae/v23n2/es_0104-1169rlae-23-02-00183.pdf

Gómez, J.J., Mayorga, M.E., Pérez, J.O., Rojas, L.Z., Orozco, L.C y Camargo, F.A. (2013). Prevalencia de diagnósticos de enfermería en personas con VIH/SIDA. Enf. Global. 32; 1-10. Recuperado de: http://scielo.isciii.es/scielo.php?pid=S1695614120130 $\underline{00400001 \& \text { script }=\text { sci_arttext }}$
Guardia, A., Lenis, P., Toro, D.C., Marín, K y Higuita, L.F. (2015). Estigma y discriminación por VIH/Sida: perspectiva de los trabajadores del sector salud y un grupo de personas que vive con la infección, Medellín, Colombia. Index 2(1); 9-22. Recuperado de: https://revistas.ucc.edu.co/index.php/cu/article/view/1 308 ( EVIDENCIA).

Hipolito, R.L., Oliveira, D.C., Costa, T.L., Marques, S.C., Pereira, E.R y Gomes, A.M.T. (2017). Calidad de vida de personas conviviendo con HIV/SIDA: relación temporal, sociodemográfica y perceptiva de la salud. Rev. Latino-Am. Enfermagem. 25; 28-74. Recuperado de: http://www.scielo.br/pdf/rlae/v25/es 0104-1169rlae-25-2874.pdf

Infante, C., Zarco, A., Cuadra, S.M., Morrison, K., Caballero, M., Bronfman, M y Magis, C. (2006). El estigma asociado al VIH/SIDA: el caso de los prestadores de servicios de salud en México. Salud Pública de México. 48 (2); 141-150. Recuperado de: http://www.redalyc.org/articulo.oa?id=10648206

Leite, R., Oliveira D.C., Costa, T.I., Corrêa, S., Ramos, E y Tosoli, A.M. (2017). Calidad de vida de personas conviviendo con HIV/SIDA: relación temporal, sociodemográfica y perceptiva de la salud. Rev. Latino-Am. Enfermagem. 25; 28-74. Recuperado de: http://www.scielo.br/pdf/rlae/v25/es_0104-1169-rlae25-2874.pdf

Ley Federal para Prevenir y Eliminar la Discriminación. DOF. 11-06-2003. Última Reforma DOF. 20-03-2014. Recuperado

de: http://www.diputados.gob.mx/LeyesBiblio/pdf/262_0 11216.pdf

Naranjo, J.M y Rodríguez, E.O. (2016). Políticas Públicas para la prevención del VIH/SIDA en adolescentes del Sector Salud. Ciencia y Tecnología.12(4); $69-82$. Recuperado http://dspace.unitru.edu.pe/handle/UNITRU/5902 (EVIDENCIA).

Norma Oficial Mexicana NOM-010-SSA2-2010. Para la prevención y el control de la infección por virus de la inmunodeficiencia humana. Recuperado de: http://www.censida.salud.gob.mx/descargas/drhuman os/NOM-010-SSA2-2010.pdf

Oliva, D., Lastre, D., Viñas, A.L y González, C. (2013). Caracterización de pacientes con abandono de la terapia antirretroviral y consecuencias económicas. Boyeros. Rev. Habanera de Cien Médicas. 14 (5); 587 598. Recuperado de: http://scielo.sld.cu/scielo.php?script=sci_arttext\&pid= $\underline{\text { S1729-519X2015000500006 }}$ 
Organización de las Naciones Unidas contra el virus de inmunodeficiencia Humana, (2016). Hoja informativa - Últimas estadísticas sobre el estado de la epidemia de sida. Recuperado de: http://www.unaids.org/es/resources/fact-sheet

Organización de las Naciones Unidas contra el virus de inmunodeficiencia Humana. (2016). Acción acelerada para acabar con el sida. Recuperado de: http://www.unaids.org/sites/default/files/media asset/ UNAIDS-strategy-2016-2021_es.pdf

Orozco, E., Alcalde, J.E., Ruiz, J.A., Sucilla, H y García, R. (2015). Mapeo político de la discriminación y homofobia asociadas con la epidemia de VIH en México. Salud Pública de México. 57(2); 190-196. Recuperado de: http://www.redalyc.org/articulo.oa?id=10639892013

Pantoja, A.L y Estrada, J.H. (2014). Discriminación y estigmatización de las personas viviendo con VIH/ SIDA: revisión de la literatura. Acta Odontológica Colombiana. 4(1); 57-80. Recuperado de: http://www.revistas.unal.edu.co/index.php/actaodonto $\underline{\text { col }}$

Programa Sectorial de Salud 2013-2018. (2013). Recuperado de: http://www.conadic.salud.gob.mx/pdfs/sectorial_salu d.pdf

Rangel, Y. (2015). Narrativas del riesgo respecto del VIH/sida en México. De letal a crónica y del estigma a los derechos humanos. Revista de El Colegio de San Luis.5(9); 200-219. Recuperado de: http://www.redalyc.org/articulo.oa?id=426240376009

Scher, A. (2016). Estigma y discriminación hacia hombres que tienen sexo con otros hombres (HSH) y mujeres trans: el impacto en la vulnerabilidad y riesgo frente al VIH/ SIDA. Study Project (ISP) Collection. 2461. Recuperado http://digitalcollections.sit.edu/isp collection/2461

Tamayo, B., Macías, Y., Cabrera, R., Henao, J.N y Cardona, J.A. (2015). Estigma social en la atención de personas con $\mathrm{VIH} /$ sida por estudiantes y profesionales de las áreas de la salud, Medellín, Colombia. Rev Cienc Salud. 13(1); 9-23. Recuperado de: http://www.scielo.org.co/pdf/recis/v13n1/v13n1a02.p $\underline{\mathrm{df}}$

Torres, A. (2006). Nuevos retos y oportunidades en un mundo globalizado: análisis político de la respuesta al VIH/ Sida en México. História, Ciências, Saúde Manguinhos. 13(3); 649-674. Recuperado de: http://www.redalyc.org/articulo.oa?id=386137991006
Varas, N y Toro, J. (2003). Políticas públicas relacionadas con el VIH/SIDA: tensión entre las necesidades individuales y colectivas. Una agenda de trabajo para la psicología en América Latina. Revista Latinoamericana de Psicología. 35(2); 195-206. Recuperado de: http://www.redalyc.org/articulo.oa?id=80535207

Varas, N., Torsten, N., Ramos, G y Cintrón, F. (2008). Desarrollo de la Escala sobre el Estigma Relacionado con el VIH/SIDA para Profesionales de la Salud mediante el uso de métodos mixtos. Revista Puertorriqueña de Psicología. 19; 183-215. Recuperado de: http://www.redalyc.org/articulo.oa?id=233216360008 